\section{Reaction times and error rates for judging nominal identity of letter strings}

\author{
DONALD BAMBER* \\ V.A. Hospital, St. Cloud, Minnesota 56301
}

Most theories of "same".s"different" judgments predict that "same" responses should be at least a slow as "different" responses. However, the contrary has often been found. To explain this, a two-processor model has been proposed. In this model, a fat processor and a slow processor operate simultaneously. "Same" responses are initiated by whichever processor first indicates that the stimuli are "same," whereas "different" responses are initiated only by the slow processor. In the experiment reported here, Ss judged whether two successively presented letter strings were nominally "same" or "different." It was expected that the fast processor would be incapable of making nominal identity judgments. Thus, both "same" and "different" responses would be initiated by the slow processor. Consequently, "same" responses should have been slower than "different" responses. However, this did not occur. This finding casts doubt upon, but does not disprove, the two-processor model.

How do people decide whether two multidimensional stimuli are "same" or "different"? Egeth (1966) proposed that people make such decisions by examining each stimulus dimension and judging whether the two stimuli are "same" or "different" (i.e., agree or disagree) with respect to that dimension. Thus, a separate judgment must be made for each stimulus dimension. These judgments may be termed elementary judgments (EJ). Egeth proposed two different ways in which the timing of these Eds might be regulated. First, all Eds might be performed concurrently and independently of each other. Although all EJs would begin simultaneously, they would not necessarily end simultaneously. Second, the Eds might be performed one at a time, one after another. Models which make the first or second aesumption may be termed parallel or serial models, respectively.

More recently, a third type of model for the timing of the Eds has been suggested by Atkinson, Holmgren, and Juola (1969), Townsend (1969, in press), and Corcoran (1971, Chap. 3). In this type of model, the LJs are performed concurrently but not independently of each other. The rate at which an $\mathrm{EJ}$ proceeds is inveruely proportional to the number of $\mathrm{F}$ is in operation at that moment. This type of model makes many of the same prediction as do serial models (Townend, 1969, in press). For purposes of this paper, this third type of model may be regarded

* The author is indebted to Raymond $O$. Collier, Jx., and Rod Rose for generouly providing computer time and helping with the proeramming. The author also wishes to the programming. The author also wishe to Vincent Stephens, Cheryl Lommel, Bea vost, and John Swenson for their aristance. as predictively equivalent to the serial models. Any remarks made regarding the predictive success or failure of serial models will also be applicable to this type of model.

When two multidimensional stimuli are "same," all EJs must be completed before $S$ can conclude that the two stimuli are "same." What about the occasions when the two stimuli are "different"? As soon as any EJ has been completed and has indicated that the two stimuli do not match with respect to that dimension, $s$ has enough information available to initiate a "different" response. The S may either initiate a "different" response at that time or he may wait until all Eds have been completed. Models that assume the former may be termed self-terminating models, whereas those that assume the latter may be termed exhaustive models.

Thus, by choosing between serial vs parallel and between self-terminating vs exhaustive, four different model types may be generated. These models may be termed one-processor models in order to differentiate them from the two-processor models which will be considered below.

A variety of predictions regarding the reaction times (RT) of "same"." "different" judgments of multidimensional stimuli may be derived from these models. Suppose it is assumed that, first, the speod of an EJ is not affected by whether the two stimuli agree or disagree with respect to that dimension and, second, S's two hands, which make the "same" response and the "different" response, are equally fast. These assumptions may be termed the isochronality assumptions. Given the isochronality assumptions, all of the one-processor models considered above predict that "same" reponses should be as slow as or slower than "different" responses.

Egeth (1966) and Nickerson (1967) did experiments to test the RT predictions of the one-processor models. They found that the "different" response RTs were in agreement with the predictions of a self-terminating model of either the serial or parallel variety. However, responses to "same" stimuli were faster than responses to stimuli which differed with respect to only one dimension. This was quite an unexpected finding. It may be termed the phenomenon of fast "same" responses. This finding indicates that, unless the isochronality assumptions are false, all of the one-processor models considered above are incorrect.

Since Egeth's and Nickerson's experiments, a large number of experiments dealing with RTs for "same"-"different" judgments have been performed. While some of these experiments did not encounter the phenomenon of fast "same" responses (Bindra, Donderi, \& Nishisato, 1968), many experiments did encounter that phenomenon (Nickerson, 1970).

Bamber (1969a) performed an experiment to test the one-processor models described above. In this experiment, the Ss were successively presented with two strings of letters and were required to indicate whether the two strings were "same" or "different." In applying the one-processor models for multidimensional discrimination to this experiment, each letter in a string was considered to be a separate stimulus dimension. Thus, an EJ consisted of comparing a letter from one string with the corresponding letter from the other string. It was found that "different" response RTs were predicted fairly well by a serial self-terminating model. However, the "same" response RTs were quite fast and were inconsistent with this model. Moreover, even when the isochronality assumptions were abandoned, the "same" RTs were still inconsistent with the serial self-terminating model.

To account for these findings, Bamber proposed a two-proceasor model. In this model, a fast processor and a slow processor simultaneously compare the two letter strings. The operation of the slow processor is described by a serial self-terminating model. The fast processor's manner of operation was never fully specified. It is assumed that the fast processor can indicate only that the two letter strings are "same." When the letter strings are "different," there is no response from the fast processor. Now, when the letter strings are "same," this is indicated by both processors. However, the fast processor usually indicates this before the slow 


\section{d f h k $1 \mathrm{~m}$ \\ $n r s t v x$} CSs.

Fig. 1. Letters used to construct the

processor. Therefore, the "same" responses are generally initiated by the fast processor. When the strings are "different," only the slow processor indicates that fact. Thus, the "different" responses are always initiated by the slow processor. This model accounts for why the "different" RTs were consistent with a serial self-terminating model, whereas the "same" RTs were faster than predicted by that model. Somewhat similar two-processor models have also been proposed by Tversky (1969), Marcel (1969), and Krueger (1970).

Physical and Nominal Identity

The fast processor's mode of operation is not known. However, it is intuitively appealing to hypothesize that the fast processor compares visual images of the two stimuli. When the images of the two stimuli match, the fast processor initiates a "same" response. When the images differ, there is no output from the fast processor.

If this hypothesis is correct, then a good method of testing the two-processor model would be to require $S$ s to judge whether or not two rows of letters were nominally identical. Two letters are considered to be physically identical if they have the same appearance and to be nominally identical if they have the same name (Posner \& Mitchell, 1967). Thus, A and $A$ are physically and nominally "same"; A and a are physically "different" but nominally "same"; and $A$ and $B$ are physically and nominally "different." Similarly, two letter strings are considered to be physically (nominally) identical if the corresponding letters from the two strings are physically (nominally) identical. So, according to the above hypothesis, the fast processor responds to letter strings which are physically "same" but does not respond to letter strings which are physically "different."1

In the experiment reported by Bamber (1969a), the two letter strings judged by $S$ were either physically and nominally "same" or physically and nominally "different." However, in the experiment reported here, the two letter strings were always physically "different"; they were to be judged" "same" or "different" according to whether they were nominally "same" or nominally "different." In order to differentiate these two experiments, let the former be termed the physical identity (PI) experiment and the latter be termed the nominal identity (NI) experiment.

Since the two letter strings were always presented successively, the string presented first may be termed the criterion string (CS) and the string presented second may be termed the test string (TS). In the NI experiment, the CS letters were typewritten and the TS letters were offset printed, using a specially constructed typeface. Thus, the CS and TS were always physically "different." Moreover, each letter in the TS could be either uppercase (UC) or lowercase (LC), independently of whether the other TS letters were UC or LC. The letters used to construct the CSs and the TSs are presented in Figs. 1 and 2, respectively.

If the hypothesis that the fast processor responds only to physically identical stimuli is correct, then it would be pointless for $S$ to employ the fast processor in the NI experiment. What about the slow processor? Assume that the slow processor's EJs, which consist of comparing a letter from the CS with the corresponding letter from the TS, may be adapted to the requirements of the task at hand. In the PI experiment, the EJs would consist of judging whether two letters were physically "same" or "different"; in the NI experiment, the EJs would consist of judging whether two letters were nominally "same" or "different." Thus, in the NI experiment, $S$ would employ the slow processor with appropriate EJs but would not employ the fast processor. Now, the operation of the slow processor is described by the serial self-terminating model (Bamber, 1969a). Consequently, the results of the NI experiment should be consistent with the serial self-terminating model. In particular, "same" responses should be slower than "different" responses. If obtained, such a result would demonstrate the empirical dissociation of the fast and slow processors and, thus, provide strong support for the two-processor model.

\section{METHOD}

Procedure

On each trial, $S$ inspected the CS outside the tachistoscope for as long as he wished. Then he rested the fingers of each hand on the two response keys and looked inside the tachistoscope. Upon being signaled "ready" by $E$, the $S$ fixated his eyes and pressed a footswitch. Two hundred milliseconds later, the TS appeared in the stimulus field for $100 \mathrm{msec}$. The S's instructions were to press the "same" key if the CS and TS were nominally identical and to press the "different" key otherwise. After pressing one of the keys, S reported verbally whether he believed his manual response had been correct or incorrect. He then received feedback concerning the accuracy of his response but not concerning its speed. The Ss had been instructed to maintain a low error rate. Within that constraint, they were told to respond as quickly as possible. Reaction time was measured to the nearest millisecond from the onset of the TS to the manual response.

It was desired that $\mathbf{S}$ should remember the CS with sufficient clarity to be able to respond to the TS quickly and accurately. It was felt that S could judge better than $E$ the amount of CS inspection time needed to achieve this level of performance. For this reason, $\mathrm{S}$ was allowed to inspect the CS for as long as he wanted. This procedure had been followed in two previous experiments (Bamber, 1969a; Bamber \& Paine, 1972 ) and had worked quite well; the Ss in these experiments had reasonably fast RTs and low error rates. Since there was a temporal gap between when $S$ viewed the CS outside the tachistoscope and the TS inside the tachistoscope, it might be feared that $S$ would forget the CS in the interval (Posner \& Keele, 1967). However, Ss are able to generate and rehearse visual images over a time interval (Posner, Boies, Eichelman, \& Taylor, 1969; Tversky, 1969). From the introspective reports of the $S s$ in the NI experiment, it appears that they did generate and/or rehearse images of the CS before viewing the TS.

\section{Subjects}

It was desired that no $\mathbf{S}$ who was incapable of maintaining an error rate of less than $3 \%$ be allowed in the experiment. Consequently, all prospective Ss were given a screening test to evaluate whether, with
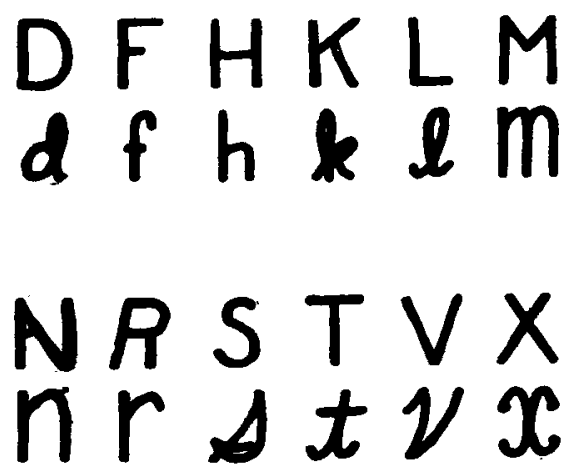

Fig. 2. Letters used to construct the TSs. Within each vertical pair of letters, the upper letter is the one designated UC. 
subsequent practice, they would be able to attain that low an error rate. Four prospective Ss were screened, and it was judged that the error rates of all four would be satisfactory. All four Ss were male undergraduates from a nearby college. All were right-handed except S 2. "Same" responses were indicated with the dominant hand by $S 2$ and $S 3$ and with the nondominant hand by $S 1$ and $S 4$. All Ss were paid volunteers.

\section{Experimental Design}

Each $S$ participated in a screening session followed by a practice session and eight test sessions lasting $11 / 2 \mathrm{~h}$ each. Each test session consisted of 12 practice trials followed by 248 test trials. On each trial, the CS and the TS contained the same number of letters. Let $L$ denote this number and let $D$ denote the number of letters in the TS which differed from the corresponding letter in the CS. In each test session, L took on the values $1,2,3$, and 4 on $48,64,72$, and 64 trials, respectively. For each value of $L$, the $C S$ and the TS were nominally "same" on half the trials, were "different" with respect to one letter $(D=1)$ on one-quarter of the trials, and were "different" with respect to every letter $(D=L)$ on the remaining quarter of the trials. Of course, when $L=1, D=1$ and $D=L$ are identical conditions. On trials when $D=1$, the discrepant letter occurred in all positions with equal frequency. Each letter in the TS could be either UC or LC. Let a specification of the case of each letter in the TS be termed the TS's case pattern. Thus, for each value of $L$, there were $2^{L}$ possible case patterns. Within every experimental condition, all possible case patterns occurred with equal frequency. Within each session, trials of all types were randomly mixed together, so that $S$ could never predict such thing as the case pattern of the TS, whether the TS would be "same" or "different," etc.

\section{Stimuli}

Both the CS and the TS consisted of a horizontal string of letters. These letters were drawn from a set of 12 consonants, all of which occurred with approximately equal frequency. Within a single string, no letter was ever duplicated, either physically or nominally. Whenever a letter appeared in both the CS and the TS, it occupied the same position in both strings. The CSs were typed, without spacing between letters, on an elite typewriter. For the TSs, a master set of letters was constructed and facsimiles of these letters were made by photo facsimiles on white cards. The TS letters were $1.9 \mathrm{~cm}$ in height. The center-to-center distance between adjacent letters in

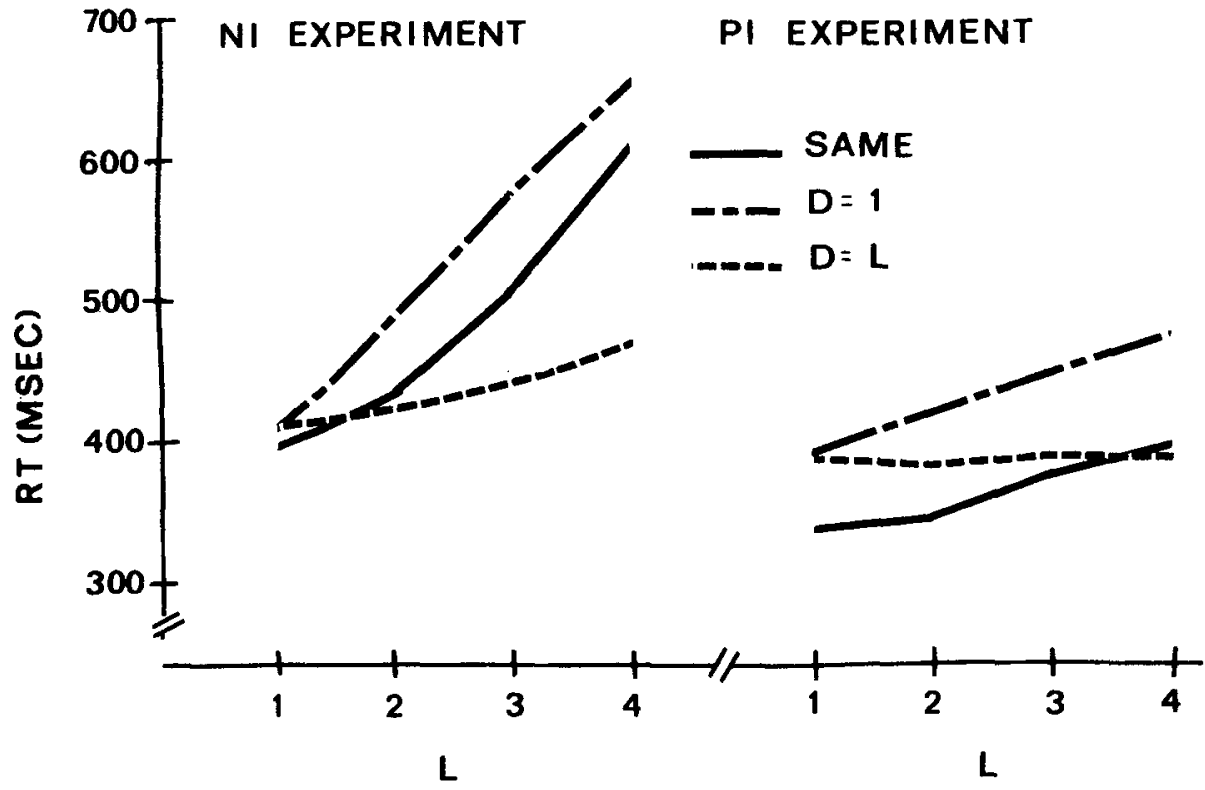

Fig. 3. Mean RTs in the "same," $D=1$, and $D=L$ conditions of the NI and PI experiments presented as functions of $L$.

the TS was $2.5 \mathrm{~cm}$ when $L=4,3.8 \mathrm{~cm}$ when $L=3$, and $5.0 \mathrm{~cm}$ when $L=2$. When viewed by $S$ in the tachistoscope, the TS appeared centered in the stimulus field.

\section{Apparatus}

The CS was presented outside the tachistoscope. The TS was exposed for $100 \mathrm{msec}$ in the stimulus field of the tachistoscope. This field measured $6 \mathrm{~cm}$ vertically $\times 10 \mathrm{~cm}$ horizontally and was viewed binocularly at an apparent distance of $100 \mathrm{~cm}$. The stimulus field was dark at the start of each trial. The fixation field was exposed continuously and consisted of two dimly lit strips $2 \mathrm{~cm}$ in height bordering the upper and lower edges of the darkened stimulus field. The Ss were instructed to fixsate the center of the dark area between the two strips. The luminances of the fixation field and the stimulus field, when exposed, were roughly $3 \mathrm{~cd} / \mathrm{m}^{2}$ and $60 \mathrm{~cd} / \mathrm{m}^{2}$, respectively. The force required to depress the response keys was roughly $25 \mathrm{~g}$.

\section{Data Analysis}

Before analyzing the RT data, a total of $1.4 \%$ of the responses were discarded: $1.1 \%$ because $S$ preased the wrong response key, $0.1 \%$ because $S$ pressed both the correct and the incorrect $\mathrm{key}$, and $0.1 \%$ because the response was slower than a cutoff value. The cutoff values for $S s 1,2,3$, and 4 were $1,200,1,600,1,200$, and $1,300 \mathrm{msec}$, respectively. Various equations were fit to the RT data, using a least-squares method in which a weighted sum of squares was minimized. The weight assigned to the mean RT of any condition was proportional to the number of trials in that condition. This method has been described in more detail elsewhere (Bamber, 1969b, App. 2). To test statistical significance, $z$ tests were employed.

\section{RESULTS AND DISCUSSION}

In reporting the results of this experiment, two conventions will be followed. First, unless stated otherwise, reported results are for the mean of the four Ss. Second, when an estimate of a quantity is stated in the form $m \pm s, m$ is the estimate and $s$ is its standard error.

\section{Best-Fit Straight Lines}

The mean RTs for the "same," $D=1$, and $D=L$ conditions are presented as a function of $L$ on the left side of Fig. 3 . Note that the "same" RTs are faster than the D=1 RTs. Thus, the phenomenon of fast "s ame" responses has been encountered once again. The RT vs $L$ graphs for the "same," $D=1$, and $D=L$ conditions appear to be fairly straight. Consequently, straight lines were fit to the three graphs. The height of a best-fit straight line at $L=1$ is referred to as its $L=1$ intercept. In the case of the $D=1$ and $D=L$ conditions, the best-fit straight lines were chosen under the constraint that the two lines intersect at $L=1$. Consequently, these two lines have a common $L=1$ intercept. The slopes of all three straight lines and their $L=1$ intercepts are presented for each $S$ in the upper portion of Table 1. All of 
Table 1

Estimates and Their Standard Errors in Milliseconds of $V$ arious Parameters of the RT vs I Graph Within Various Conditions of the NI and PI Experiments

\begin{tabular}{|c|c|c|c|c|c|c|}
\hline & \multicolumn{2}{|c|}{$\mathbf{L}=\mathbf{1}$ Intercept } & \multicolumn{3}{|c|}{ Slope } & \multirow{2}{*}{$\frac{\text { Quadratic }}{\text { "Same" }}$} \\
\hline & "Same" & "Different"' & "Same" & $\mathbf{D}=\mathbf{1}$ & $\mathbf{D}=\mathbf{L}$ & \\
\hline \multicolumn{7}{|c|}{ NI Experiment } \\
\hline $\begin{array}{l}\text { S } 1 \\
\text { S } 2 \\
\text { S } 3 \\
\text { S } 4 \\
\text { All Ss }\end{array}$ & $\begin{array}{l}343.9 \pm 3.4 \\
371.6 \pm 4.7 \\
412.8 \pm 3.8 \\
398.0 \pm 3.9 \\
381.6 \pm 2.0\end{array}$ & $\begin{array}{l}363.3 \pm 3.5 \\
388.5 \pm 4.1 \\
446.8 \pm 3.7 \\
435.6 \pm 4.3 \\
408.6 \pm 2.0\end{array}$ & $\begin{array}{r}42.8 \pm 2.6 \\
118.9 \pm 4.2 \\
60.3 \pm 2.5 \\
65.0 \pm 2.4 \\
69.3 \pm 1.5\end{array}$ & $\begin{array}{r}59.0 \pm 3.2 \\
136.4 \pm 5.4 \\
66.4 \pm 3.3 \\
60.9 \pm 3.9 \\
80.7 \pm 2.0\end{array}$ & $\begin{array}{r}7.1 \pm 1.8 \\
47.9 \pm 2.3 \\
11.9 \pm 1.9 \\
13.5 \pm 2.2 \\
20.1 \pm 1.0\end{array}$ & $\begin{array}{r}11.0 \pm 2.5 \\
27.3 \pm 4.1 \\
21.7 \pm 2.5 \\
4.7 \pm 2.6 \\
16.2 \pm 1.5\end{array}$ \\
\hline \multicolumn{7}{|c|}{ PI Experiment } \\
\hline All Ss & $330.9 \pm 1.3$ & $382.5 \pm 1.4$ & $20.0 \pm 0.6$ & $31.5 \pm 1.2$ & $2.0 \pm 0.8$ & $5.1 \pm 0.7$ \\
\hline
\end{tabular}

these slopes are significantly greater than zero. For each $S$, the slope of the RT vs $L$ graph for the $D=L$ condition is significantly less than the slopes for the "same" and $D=1$ conditions. Finally, for each $S$, the slope for the "same" condition is numerically, but not necessarily significantly, less than the slope for the $D=1$ condition. For $S 1, S 2$, and for all of the $S$ s combined, this difference is statistically significant.

The RT vs $L$ graphs for the $D=1$ and $D=L$ conditions are fairly well fit by straight lines. For each mean RT in the $D=1$ and $D=L$ conditions, the discrepancy between the observed RT and the best-fit straight line was calculated. The root-mean-square of all those discrepancies was 3.9 msec. However, the RT vs L graph for the "same" condition is not so well fit by a straight line. For the "same" condition, the root-meansquare discrepancy between the observed RTs and the best-fit straight line was 16.0 msec.

Examination of Fig. 3 shows that the RT vs L graph for the "same" condition deviates from linearity by being somewhat concave upward. To determine whether the degree of upward concavity was statistically significant, the "same" RT vs L graph was tested for the presence of a quadratic component. The component tested was the function: $\mathrm{L}^{2}-5.080 \mathrm{~L}$ + 5.307. This function was chosen because, when added to the regression equation, it does not affect the values of the previously estimated slope and $L=1$ intercept. The amount of this quadratic component in the "same" RT vs $L$ graph of each $S$ is presented in Table 1. For every $S$ except $S 4$, the size of this component is significantly greater than zero. Thus, the "same" RT vs $L$ graph is significantly concave upward.

\section{One-Processor Models}

It was predicted that, if the two-processor model was correct and if the fast processor responded only to physically identical stimuli, then the results of the NI experiment should be consistent with the serial self-terminating model. Are the results of the NI experiment consistent with this model or any of the other one-processor models discussed above? How well some of the RT predictions of these models fit the data will be considered below. It should be noted that none of the predictions considered depend on the isochronality assumptions.

Consider first the serial self-terminating and serial exhaustive models. The former model predicts that the slope of the RT vs L graph for the "same" condition should be twice as great as the slope for the $D=1$ condition; the latter model predicts that the two slopes should be equal (Sternberg, 1966). However, Table 1 shows that the slope for the "same" condition is somewhat less than the slope for the $D=1$ condition. Therefore, both these models are wrong. Moreover, the serial self-terminating model predicts that the RTs in the $D=L$ condition should be independent of $L$. However, Table 1 shows that the RT vs L graph for the $D=L$ condition has a positive slope.

Next, consider the parallel models. A proof is given in the Appendix showing that the parallel models predict that the "same" RT vs L graph should be convex upward. However, the "same" RT vs L graph is actually concave upward. Thus, the parallel models are wrong also. Note that the concavity of the "same" RT vs L graph is also inconsistent with the serial models. These models predict that the graph should be linear.

Now, all of the one-processor models discussed above implicitly assume that the Eds consist of, first, encoding a TS letter and, second, comparing the encoded TS letter with a previously encoded CS letter. However, these models may be modified by assuming that every one of the TS letters must be encoded before any one of them can be compared with its corresponding CS letter. Thus, in the modified one-processor models, the encoding of the TS letters precedes the EJs which consist of letter comparisons only.

What RT predictions do the modified one-processor models make? The predicted RTs of the modified models may be obtained by adding on to the predicted RTs of the original models the amount of time by which the commencement of the EJs is delayed by the encoding of the TS. Now the size of this delay will increase with $L$. If the TS letters are encoded serially, the amount of delay will be a linear function of $\mathrm{L}$. The proof in the Appendix shows that, if the TS letters are encoded in parallel, the amount of delay will be a convex-upward function of $\mathrm{L}$.

Are the RT predictions of any of the modified one-processor models consistent with the results of the NI experiment? As the reader can verify, the answer is: No. The only substantial improvement in the RT predictions of the one-processor models is that the modified serial self-terminating model predicts a positive slope for the $D=L$ RT vs L graph.

\section{Position Effects}

In the $D=1$ conditions, $R T$ was faster when the lone "different" letter was on the left side of the TS than when it was on the right. Graphs were constructed plotting mean RT in the $\mathbf{D}=1$ condition vs the left-right position of the "different" letter. Separate graphs were constructed for $L=2,3$, and 4 . The slopes of the best-fitting straight lines for these graphs were $20.9 \pm 6.7,23.7 \pm 5.7$, and $52.1 \pm 6.6 \mathrm{msec}$, respectively. The corresponding slopes were also calculated for individual Ss. All of the slopes for individual Ss were positive, half of them significantly so.

\section{Case Effects}

It was desired to see whether the case of the letters in the TS had any effect on RT. For each condition in the experiment, a graph was made plotting mean RT vs the number of LC letters in the TS. If the TS contained a total of L letters, the number of LC letters could vary from zero to $L$. For each of these graphs, the slope of the best-fitting straight line was calculated. The values of these slopes are presented in Table 2. In the "same" conditions, the slopes are all significantly greater than zero and appear to be independent of $L$. However, in the "different" conditions, while all the slopes are positive, only one is significantly greater than zero. Moreover, the values of these slopes vary erratically with $L$. Thus, at least for "same" TSs, UC letters tend to be processed faster than LC letters. 


\section{Errors}

The overall error rate in this experiment was $1.1 \%$. However, this error rate was not uniform across all conditions. The error rate in each condition of the NI experiment is presented on the left side of Fig. 4. Not only was the overall error rate low, but Ss were also usually aware of when they had made an error. On every trial, after pressing either the "same" key or the "different" key, the $S$ reported verbally whether he believed his manual response had been correct or incorrect. On $90 \%$ of the trials when the $S$ made an error, he reported that his response had been in error. In the entire experiment, only one trial occurred where an $S$ reported that he had made an error when, in fact, he had pressed the correct key.

\section{Comparison with}

the PI Experiment

Once the data from the NI experiment had been analyzed, it became clear that these results bore a striking resemblance to the results of the PI experiment (Bamber, 1969a). A comparison of the results of these two experiments is presented here. It should be noted, however, that precise comparisons of the NI and PI experiments cannot be made because different $S$ s were used in the two experiments and because the experiments were not of equal duration.

The mean RTs from the "same," $D=1$, and $D=L$ conditions of the $P I$ experiment are presented on the right side of Fig. 3. The intercepts, slopes, and quadratic component that were computed for the NI experiment were also computed for the PI experiment and are presented in the bottom line of Table 1. The quadratic component tested in the PI data was the function: $\mathrm{L}^{2}-5.188 \mathrm{~L}+5.410$. Again, this function was chosen because it did not affect the values of the previously estimated slope and intercept. The error rates for various conditions of the PI experiment are presented on the right side of Fig. 4.

Examining the results presented in Figs. 3 and 4 and Table 1 shows that, although RTs in the PI experiment are

Table 2

Estimates and Their Standand Frrors in Millieconds of the Slope of the Gmph Plotting Mean RT vs the Number of LC Letters in the TS Within Each Condition of the NI Experiment

\begin{tabular}{lcrr}
\hline L $=$ & "Same" & D $=1$ & \multicolumn{1}{c}{ D = L } \\
\hline 1 & $12.2 \pm 4.6$ & $2.1 \pm 4.9$ & \\
2 & $10.2 \pm 3.1$ & $3.9 \pm 5.2$ & $10.4 \pm 3.8$ \\
3 & $11.4 \pm 3.4$ & $10.9 \pm 7.0$ & $5.1 \pm 3.3$ \\
4 & $14.4 \pm 4.8$ & $8.3 \pm 7.8$ & $3.4 \pm 2.7$ \\
\hline
\end{tabular}

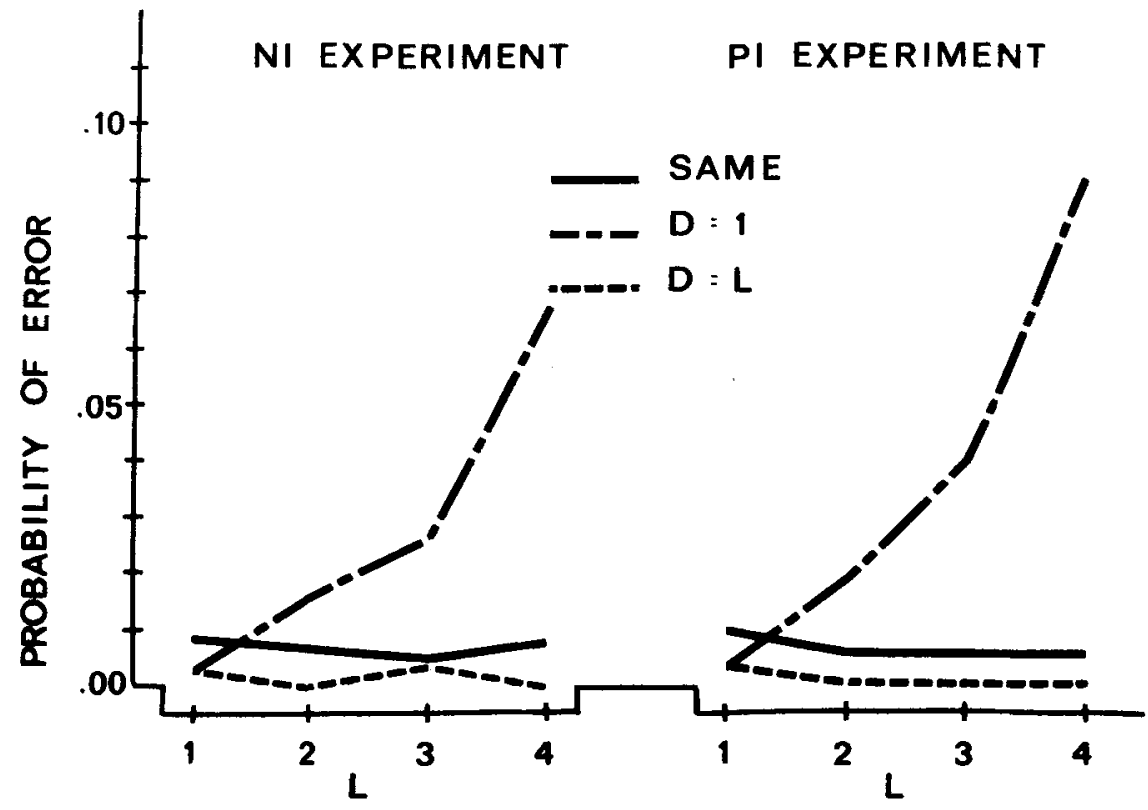

Fig. 4. Error rates in the "same," $D=1$, and $D=L$ conditions of the $N I$ and PI experiments presented as functions of $L$.

faster than those in the NI experiment, there are a number of similarities between the results of the two experiments. Now, as discussed above, Egeth (1966) proposed that people decide whether multidimensional stimuli are "same" or "different" by combining a number of EJs. The results of the NI and PI experiments suggest that the Eds were combined using the same method in both experiments, but that each EJ took more time in the NI experiment than in the PI experiment.

There are two problems with this explanation. First, although the EJs may have been combined using the same method in both experiments, it is not known what that method is. Second, although significantly greater than zero in both experiments, the slope of the RT vs $L$ graph for $D=L$ is virtually negligible in the PI experiment, but quite substantial in the NI experiment.

Why would the EJs in the NI experiment be slower than those in the PI experiment? People are able to generate visual images (Posner et al, 1969; Tversky, 1969). Now, the Ss in the NI experiment all claimed that they formed some kind of visual representation of the CS before viewing the TS. This suggests two different methods that an $\mathrm{S}$ in the NI experiment might use to compare a CS letter with a TS letter. First, given a TS letter as illustrated in Fig. 2, S would generate an image of the corresponding letter in Fig. 1 . This image would then be compared with the CS letter. Second, given a CS letter as illustrated in Fig. $1, S$ would generate images of the corresponding UC and LC letter in Fig. 2. These two images would then be compared with the TS letter. Such generation of letter images would not be necessary in the PI experiment. Consequently, EJs would be slower in the NI experiment. 2

\section{Comparison with Other Experiments}

Eichelman (1970, Experiment I) and Beller (1970, Experiment II) performed experiments involving "same"."ddifferent" judgments of letter strings. In both these experiments, the two strings were presented simultaneously, one above the other, rather than successively as in the PI and NI experiments.

In Eichelman's experiment, each $\mathbf{S}$ judged whether two words or two random letter strings were physically identical. Only the latter condition will be considered here. From Figs. 1 and 2 of Eichelman's article, the slope of the RT vs L graph was estimated for the "same," $D=1$, and $D=L$ conditions. These slopes were roughly 60,93 , and 15 msec, respectively. For $L=1$, "same" RTs were about 30 msec faster than "different" RTs. Unlike Bamber's PI experiment, the slope in the $D=L$ condition was not negligible. Otherwise, the results from the two experiments were similar.

In Beller's experiment, the $S$ s judged whether the two strings were nominally identical. Unlike the NI experiment reported here, corresponding letters from the two strings could be physically identical. The slope of the RT vs $L$ graph in the $\mathrm{D}=1$ and $\mathrm{D}=\mathrm{L}$ conditions was 
estimated from Table 5 of Beller's article. These slopes were roughly 86 and 22 msec, respectively. These values are similar to those obtained in the NI experiment. In the "same" condition, the slope of the RT vs $L$ graph varied with the number of physically identical letter pairs contained in the two strings. Depending on whether the number of such pairs was $L, L-1$, or zero, the slope of the "same" RT vs L graph was 63,61 , or 143 msec, respectively. Unfortunately, Beller did not state how many physically identical letter pairs the two strings contained in the $\mathbf{D}=1$ condition. Thus, it is not clear how to make an appropriate comparison between the slope in the $\mathrm{D}=1$ condition and the slopes in the "same" condition.

\section{Status of the}

Two-Processor Model

The present experiment and another experiment (Bamber \& Paine, 1972) were designed to "turn off" the fast processor and, thus, dissociate the two processors of the two-processor model. In both of these experiments, "same" responses should have been slower than "different" responses. However, this did not occur in either case. These results cast doubt upon the two-processor model. The failure of these experiments to dissociate the two processors suggests that there may be only one processor. On the other hand, none of the one-processor models that were considered fit the data.

There are two ways out of this dilemma. One is to search for new one-processor models. The other is to assume that the two-processor model is correct but that the fast processor is more robust and adaptable than had been previously supposed. However, even if the two-processor model is correct, it will still be necessary to search for new one-processor models. In the two-processor model, "same" responses are initiated by the fast processor. Nevertheless, none of the one-process or models considered above could explain why the "same" RT vs L graph was concave upward.

\section{REFERENCES}

ATKINSON, R. C., HOLMGREN, J. E., JUOLA, J. F. Processing time" as influenced by the number of elements in a visual display. Perception \& Psychophysics, 1969, 6, 321-326.

BAMBER, D. Reaction times and error rates for "same"_"different" judgments of multidimensional stimuli. Perception \& Psychophysics, 1969a, 6, 169-174.

B AMBER, D. "Same" "different" judgments of multidimensional stimuli: Reaction times and error rates. (Doctorial dissertation, Stanford University) Ann Arbor, Mich: University Microfilms, $1969 b$, No. 69-17,395.

BAMBER, D., \& PAINE, S. Information retrieval processes in "same"-"different" judgments of letter strings. In $S$ Kornblum (Ed.), Attention and performance IV. New York: Academic Press, 1972, in press.

BELLER, H. K. Parallel and serial stages in matching. Joumal of Experimental Psychology, 1970, 84, 213-219.

BI NDRA, D., DONDERI, D. C., \& NISHISATO, $\mathrm{s}$. Decision latencies of "same" and "different" judgments. Perception \& Psychophysics, 1968,3 , 121-130.

CORCORAN, D. W. J. Pattern recognition. Baltimore: Penguin, 1971.

EGETH, H. E. Parallel versus serial processes in multidimensional stimulus discrimination. Perception \& Psychophysics, 1966, 1, 245-252.

EICHELMAN, W. H. Familiarity effects in the simultaneous matching task. Journal of Experimental Psychology, 1970, 86 . $275-282$.

KRUEGER, L. E. Effect of bracketing lines on speed of "same"_cdifferent" judgment of two adiacent letters. Journal of Experimental Psychology, 1970, 84, 324-330.

MARCEL, A. J. Serial and parallel processing in pattern recognition. Paper presented at the meeting of the Experimental Psychology Society, Lond on, January 1969. Cited by Nickerson (1970, pp. 159-160).

NICKERSON, R. S. "Same" "different" response times with multi-attribute stimulus differences. Perceptual \& Motor Skills, 1967, 24, 543-654.

NICKERSON, R. S. Binary-classification reaction time: A review of some studies of human information-processing capabilities. Report No. 2004, Decembex 28, 1970, Bolt Beranek and Newman, Inc. Cambridge, Mass., Contract F44620-69-C-0115, Air Force Office of Scientific Research.

POSNER, M. I., BOIES, S. J., EICHELMAN, W. H., \& TAYLOR, R. L. Retention of visual and name codes of single letters. Joumal of Experimental Psychology, 1969, 79(1, Pt. 2).

POSNER, M. I., \& KEELE, S. W. Decay of visual information from a single letter. Science, 1967, 158, 137-139.

POSNER, M I \& MITCHELL, R. F Chronometric analysis of classification. Psychological Review, 1967, 74, 392-409. STERNBERG, $S$. High-speed scanning in human memory. Science, 1966, 153 , 652-654.

TOWNSEND, J. T. Mock parallel and serial models: An observation on the methodology of model testing. Paper presented at the Mathematical
Psychologists Conference, Indiana University, 1969

TOWNSEND, J. T. Some results concerning the identifiability of parallel and serial processes. British Journal of Mathematical \& Statistical Psychology, in press.

TVERSKY, B. Pictorial and verbal encoding in a short-term memory task. Perception \& Psychophysics, 1969, 6, 225-233.

(Accepted for publication June 15, 1972,)

APPENDIX

It is desired to show that a parallel model predicts that the mean RT of a "same" response is a negatively accelerated function of $L$. Let $T$ be a random variable denoting the amount of time taken by an EJ to terminate in a "same" decision. It is assumed that $T$ is a continuous random variable. Let $T_{1}, \ldots, T_{k}$ represent $k$ observations each sampled randomly from the $T$ distribution. Let $M_{k}$ denote the maximum of $T_{1}, \cdots, T_{k}$. For any random variable $W$, let $E(W)$ denote its expected value. For any event $A$, define $I(A)$ as the random variable having the value one, if $A$ occurs, and the value zero otherwise. Define the random variables $X, Y$, and $Z$ as follows:

$$
\begin{aligned}
& X=\left(T_{L+1}-M_{L}\right) I\left(T_{L+1} \geqslant M_{L}, T_{L+2}\right) \\
& Y=\left(T_{L+2}-M_{L}\right) I\left(T_{L+2} \geqslant M_{L}, T_{L+1}\right) \\
& Z=\left(T_{L+1}-M_{L}\right) I\left(T_{L+2}>T_{L+1}>M_{L}\right)
\end{aligned}
$$

Then

$$
\begin{aligned}
& \mathbf{E}\left(\mathbf{M}_{\mathbf{L}+\mathbf{1}}\right)-\mathbf{E}\left(\mathbf{M}_{\mathbf{L}}\right)=\mathbf{E}(\mathbf{X})+\mathbf{E}(\mathbf{Z}) \\
& \mathbf{E}\left(\mathbf{M}_{\mathbf{L}+\mathbf{2}}\right)-\mathbf{E}\left(\mathbf{M}_{\mathbf{L}}\right)=\mathbf{E}(\mathbf{X})+\mathbf{E}(\mathbf{Y})
\end{aligned}
$$

So

$$
\mathbf{E}\left(\mathbf{M}_{\mathbf{L}+2}\right)-\mathbf{E}\left(\mathbf{M}_{\mathbf{L}+1}\right)=\mathbf{E}(\mathbf{Y})-\mathbf{E}(\mathbf{Z})
$$

It is evident from symmetry that $E(X)$ equals $E(Y)$. Then, since $E(Z)$ is greater than zero, it follows that $E\left(M_{L_{+1}}\right)-E\left(M_{L}\right)>$ $\mathbf{E}\left(\mathbf{M}_{\mathbf{L}+2}\right)-\mathbf{E}\left(\mathbf{M}_{\mathbf{L}+1}\right)$. This shows that $E\left(M_{L}\right)$ and, therefore, the mean $R T$ of a "same" response are negatively accelerated functions of $L$.

NOTES

1. Although this is an intuitively appealing hypothesis, it is not a logical necessity. Conceivably, the fast processor might respond to letter strings which are nominally "same" but physically "different"

2. Alternatively, the $S s$ in the NI experiment might have encoded both the CS and the TS letter auditorily and then compared the two auditory codes. However. this would not explain why the Ss claimed that they formed a visual representation of the CS before viewing the TS. Conceivably, the Ss may have followed some hybrid strategy employing both visual and auditory codes. 